Giovanni Miccoli, Les dilemmes et les silences de Pie XII. Vatican, Seconde Guerre mondiale et ShoahParis, Éditions Complexe, coll. " Histoire du temps présent", 2006, 473 p.Yves Chevalier

Paris, Éditions Complexe, coll. « Histoire du temps présent », 2006, 473 p.

\title{
OpenEdition
}

Journals

Édition électronique

URL : http://journals.openedition.org/assr/11673

DOI : $10.4000 /$ assr. 11673

ISSN : 1777-5825

Éditeur

Éditions de l'EHESS

Édition imprimée

Date de publication : 1 décembre 2007

Pagination : 157-310

ISBN : 978-2-7132-2145-3

ISSN : 0335-5985

Référence électronique

"Giovanni Miccoli, Les dilemmes et les silences de Pie XII. Vatican, Seconde Guerre mondiale et ShoahParis, Éditions Complexe, coll. «Histoire du temps présent », 2006, 473 p. Yves Chevalier », Archives de sciences sociales des religions [En ligne], 140 | octobre - décembre 2007, document 140-62, mis en ligne le 02 juillet 2008, consulté le 10 décembre 2020. URL : http://journals.openedition.org/assr/ 11673 ; DOI : https://doi.org/10.4000/assr.11673

Ce document a été généré automatiquement le 10 décembre 2020.

(C) Archives de sciences sociales des religions 


\section{Giovanni Miccoli, Les dilemmes et les silences de Pie XII. Vatican, Seconde Guerre mondiale et ShoahParis, Éditions Complexe, coll. « Histoire du temps présent », 2006, 473 p.Yves Chevalier}

Paris, Éditions Complexe, coll. « Histoire du temps présent », 2006, 473 p.

Le thème n'est pas neuf, et depuis le milieu des années 1960, c'est même un sujet de polémiques à propos duquel défenseurs et accusateurs de Pie XII s'affrontent à coups d'arguments indécidables. Giovanni Miccoli, titulaire d'une chaire d'Histoire de l'Église à la Faculté des lettres de l'Université de Trieste, a voulu ici faire œuvre d'historien : non pas essayer de percer ce que Pie XII, les membres de la Curie et, encore plus largement, les évêques des différentes Églises locales « auraient dû faire » (qu'ils n'ont pas fait) ou expliquer pourquoi ces mêmes personnages « ne pouvaient pas faire autre chose » (que ce qu'ils ont fait), mais « déterminer en premier lieu ce qui a été fait et pourquoi, à la lumière du contexte dans lequel [Pie XII] et ses collaborateurs ont dû agir, selon les idées, les attentes et les jugements qui les ont tour à tour orientés et motivés.» Travail sur archives, donc, celles du Saint-Siège lorsqu'elles étaient disponibles, celles des Églises nationales aussi, non point pour juger les comportements des uns et des autres avec ce que nous savons de la fin de l'histoire, mais pour comprendre - ce qui ne veut pas dire justifier ni absoudre - les attitudes adoptées et les décisions prises en fonction de leurs « conditions de production ». Il faut souligner la qualité et l'abondance de la documentation sur laquelle l'auteur fonde son analyse ; et ajouter qu'entre la version italienne (qui date de 2000) et la traduction française, il a modifié son texte en fonction, entre autre, de l'ouverture de certaines sections des Archives du Vatican relatives aux relations du Saint-Siège avec l'Allemagne pendant le pontificat de Pie XI (1922-1939) - non que cela l'eût obligé à revoir ses conclusions, au contraire, mais par souci de précision et de rigueur. 
2 L'ouvrage proposé cherche à examiner un segment - le rôle de l'Église catholique au sens large - significatif du contexte dans lequel la Shoah s'est produite. Il se présente comme une suite de "dossiers", chacun traitant d'un problème particulier mais interdépendant des autres. G. Miccoli ouvre d'abord son propos en écartant «deux questions non dépourvues d'ambiguïté » qui ont trop souvent servi à formuler les jugements sur l'attitude de l'Église à cette période sans faire avancer la compréhension $\mathrm{du}$ problème. Pour lui, que cette attitude traduise une carence du magistère ou une perspicacité pastorale, le résultat est le même: ily a eu défaut d'une parole que beaucoup auraient souhaité entendre. De même, la question : que savait-on de la Shoah au Vatican? est de peu d'intérêt parce que, si beaucoup d'informations étaient disponibles (le Vatican étant un carrefour où convergeait une multitude de canaux externes et internes à l'Église), ce qui compte, c'est de pouvoir - et de vouloir - les transformer en connaissances susceptibles d'orienter l'action.

3 Le premier chapitre traite du "Saint-Siège et la Seconde Guerre mondiale », et de la difficile élaboration d'une ligne politique claire. Reprenant, malgré son échec patent lors de la Première Guerre mondiale, la position définie par Benoît XV à propos des conflits entre États et de la neutralité nécessaire du siège apostolique, Pie XII se trouva, dès l'éclatement de la guerre (qu'il avait, comme son prédécesseur Pie XI, tenté d'éviter), enfermé dans une situation inextricable : il ne concevait son rôle que comme celui d'un arbitre, qui plus est un arbitre disposant de lumières spéciales susceptibles d'éclairer l'humanité et les parties en conflit sur la voie à prendre pour sortir de l'impasse. C'est ce qu'il dit dans sa première encyclique, Summi pontificatus, du 20 octobre 1939, et que l'on retrouve dans d'autres discours de la même époque. Or une telle position d'équilibre (qui ne lui interdisait nullement d'intervenir, ce qu'il fit à de nombreuses reprises, mais seulement " diplomatiquement ", c'est-à-dire à travers toute une série d'intermédiaires) est devenue un obstacle pour réagir face à la «guerre totale » et aux comportements nazis dans les territoires occupés, mais aussi face à la politique des conversions forcées menée dans l'État indépendant de Croatie. C'est une position similaire qui semble avoir guidé l'action du Saint-Siège en ce qui concerne le problème juif: l'action diplomatique - à travers nonces et délégués apostoliques semble s'être essentiellement tournée vers les pays alliés de l'Allemagne pour prêcher la modération et intervenir indirectement dans l'aide aux persécutés; et l'attitude de méfiance du Saint-Siège envers les juifs, héritage du traditionnel antijudaïsme de l'Église, n'a fait que paralyser un peu plus le Pape et les autorités de la Curie devant des faits connus mais qu'ils ne semblent pas avoir analysés à leur exacte portée - et en tout cas, dont ils n'ont pas compris qu'ils mettaient en cause le christianisme lui-même. Un corollaire de cette position de neutralité bienveillante est donné dans la dernière section de ce chapitre, qui traite des relations entre Pie XII et l'épiscopat allemand, et que Giovanni Miccoli définit comme " un équilibre précaire entre parole et silence », le Pape préférant laisser intervenir l'Église locale, c'est-à-dire les Évêques et la Conférence épiscopale, considérés comme plus à même d'apprécier la situation et les possibilités d'action.

4 Le deuxième chapitre, en prolongement, aborde la question du catholicisme allemand et du national-socialisme. Il convient ici de remonter plus haut, jusqu'au début des années 1930 et même avant, car la mémoire du Kulturkampf est un facteur qui joue encore. Ce qui apparaît bien, c'est que la rencontre entre l'Église allemande et le national-socialisme fut fragile et contraignante - la signature d'un concordat, que 
certains avaient considéré comme un moyen de mieux maîtriser la situation, s'avérant à l'usage plus néfaste qu'autre chose. Le déséquilibre entre l'accroissement de la persécution religieuse d'une part, la confirmation du loyalisme ecclésiastique d'autre part, ne fit que s'accentuer, même si, du côté de l'épiscopat allemand, des appréciations plus nuancées avaient pu apparaître. Ici, la position de Pie XI qui, sur la fin de sa vie, envisageait de plus en plus la nécessité d'une rupture, diverge de celle de Pie XII, enclin à de nouveaux compromis - même si, là aussi, il convient de ne pas durcir les oppositions, certaines prises de position de Pie XI ayant été préparées par le cardinal Pacelli; et une véritable continuité de pensée et de principe d'action est perceptible entre les deux. Il reste que l'éclatement de la guerre, les victoires fulgurantes de l'Allemagne dans un premier temps puis le reflux des armes ensuite, compliquèrent encore davantage la vie interne de l'Église d'Allemagne, qui fut loin de pouvoir définir une position commune face à la guerre et face au pouvoir politique; accusé par les nazis de défaitisme et de manque de solidarité avec la nation, l'épiscopat en fut réduit à recommander, de manière un peu contradictoire, l'accomplissement loyal de devoir contre l'ennemi extérieur et simultanément le repli intérieur dans l'affirmation et la défense des droits de l'Église.

Les deux chapitres suivants: "Le Vatican et le péril communiste » et «Le Vatican et l'occupation allemande de Rome", plus courts, traitent de deux questions connexes, mais dont l'importance n'est pas à négliger pour comprendre la politique globale du Saint-Siège pendant cette période. La première, la question de la peur du communiste, parce qu'il s'agit d'une vieille inimitié, certes fondée sur une expérience mais quelque peu fantasmée; surtout lorsqu'elle conduisait certains à préférer la victoire de l'Allemagne nazie à celle de l'URSS de Staline. Mais il est certain que pour Rome, et pour Pie XII en particulier, une victoire communiste aurait été une catastrophe; et cette considération explique les efforts du Pape pour épargner l'Allemagne et obtenir des Alliés un accord séparé - et antisoviétique - avec elle. Quant à l'occupation allemande de Rome, après la chute de l'État italien, elle ne fera que renforcer la «prudence » et la « réserve» du Vatican. Directement confronté aux « vicissitudes des conflits terrestres » et surtout à la rafle des juifs de Rome dans la nuit du 15 au 16 novembre 1943, Pie XII maintient sa ligne de conduite constante: ne pas rompre avec les autorités allemandes, éventuellement menacer d'une action publique pour essayer d'obtenir concessions et atténuations des mesures.

Reste l'examen de la position du Saint-Siège et de l'opinion catholique face à l'antisémitisme et aux lois raciales. C'est pour l'auteur l'occasion de revenir sur l'antijudaïsme et sur l'antisémitisme de l'Église au xix et au début du $\mathrm{xx}^{\mathrm{e}}$ siècle, avant d'étudier «L'Église allemande et la persécution des Juifs en Allemagne dans les années trente » - où il met en évidence que la passivité des catholiques allemands et de la hiérarchie catholique (mais cela est vrai aussi pour l'Église évangélique et ses fidèles) est largement due à cette imprégnation d'un antijudaïsme théologique (même s'il remarque qu'il convient de se méfier des causalités automatiques en histoire). Il est certain qu'on trouve là l'un des arguments utilisés par les nazis pour empêcher toute velléité de réaction : «nous ne faisons que continuer ce que vous-mêmes vous avez fait pendant des siècles». Ce "réflexe» antijuif, qui n'est nullement le propre des catholiques allemands, est aussi l'une des dimensions qui aident à comprendre la réaction - ou l'absence de réaction - des catholiques devant les lois antijuives en France 
ou en Italie, tout autant que dans les milieux du Saint-Siège où les législations spéciales sont tolérées, à moins qu'elles ne dépassent certaines limites très théoriques.

7 Pourtant, on sait que Pie XI, à la fin du mois de juin 1938, avait chargé un jésuite, le père La Farge, en lui exposant « le thème dans ses grandes lignes, la méthode à suivre et les principes à respecter ", de réfléchir à une encyclique condamnant l'antisémitisme. Le projet, auquel travaillent, à Paris, La Farge et deux puis trois de ses confrères, est prêt à la fin de l'été et remis au Père Général des Jésuites fin septembre, pour n'arriver - manifestement à sa demande - sur le bureau du Pape que le 21 janvier 1939, à un moment où, déjà malade, il n'est plus en mesure d'y travailler. Pie XI meurt le 10 février. Il est donc impossible de savoir ce qu'il aurait fait de ce projet, si celui-ci a été remis à son successeur - il semble que ce fut le cas - et dans cette dernière hypothèse les raisons qui l'ont conduit à ne pas reprendre à son compte le projet de son prédécesseur. Il est vrai que le projet allait beaucoup plus loin que Mit brennender Sorge, en ce qu'il condamnait directement le racisme et l'antisémitisme (bien que sur ce dernier point, sur l'attitude de l'Église face aux persécutions, sur la manière dont il tente de situer la «question juive » dans une perspective strictement théologique, le texte soit nettement "pré-conciliaire »). L'auteur, contrairement à d'autres, regrette que ce projet n'ait pas abouti, à la fois parce que « la condamnation de l'antisémitisme nazi était exprimée en des termes qui ne furent jamais utilisés ni avant ni après » et parce que « la publication du texte aurait en tout cas offert une référence officielle et capitale à l'opinion publique catholique pour se dissocier de l'antisémitisme et s'y opposer. »

8 En l'absence d'une telle prise de position officielle, qui aurait cependant, c'est tout le livre de Giovanni Miccoli qui le montre, été à l'encontre de toute la tradition diplomatique vaticane et même de la façon de penser des responsables de la Curie, les choses reprirent leur cours, et c'est la voie diplomatique qui fut privilégiée : l'auteur en donne de multiples exemples en suivant successivement «le Saint-Siège et la persécution des juifs en Italie au cours de la guerre ", "L'Église, le gouvernement de Vichy et la persécution des juifs " (et la fameuse consultation de l'ambassadeur Léon Bérard auprès des " autorités de Vatican » au sujet du Statut des juifs), «L'Épiscopat allemand face à la Shoah ", "Le Saint-Siège et la Shoah dans l'Europe orientale : la Slovaquie, la Hongrie et la Croatie ». Une section particulière aborde «la question des représailles nazies à la lettre pastorale des évêques hollandais " et discute le motif exact de ces représailles.

De cette longue étude documentée, et dans une courte conclusion, Giovanni Miccoli en arrive à mettre en évidence que « les conditionnements d'une tradition idéologique et diplomatique » constituent le facteur essentiel qui explique pourquoi le Vatican, et en particulier Pie XII, semblent n'avoir pas véritablement saisi la situation créée par l'apparition sur la scène internationale d'un régime comme celui d'Hitler - ou s'ils l'ont partiellement saisi, n'ont pas été capables d'imaginer une autre attitude que celle qu'ils ont effectivement adoptée et qui nous paraît, à nous, inadéquate. Il s'agit là d'un problème de mentalité, d'« anachronisme » dit l'auteur, qui fait que les acteurs furent incapables d'évaluer à sa juste mesure l'écart entre la réalité et le discours. 